\title{
Entrevista a Joan Pagès Blanch
}

\author{
Natalia Contreras Quiroz'
}

Joan Pagès es Licenciado en Filosofía y Letras e Historia Moderna y Contemporánea. Doctor en Ciencias de la Educación (UAB, 1993) y profesor de secundaria. Actualmente es profesor catedrático de asignaturas de Didáctica de las Ciencias Sociales en la Universidad Autónoma de Barcelona. Con una experiencia como profesor que supera las cuatro décadas, ha desarrollado también investigaciones en las áreas de la formación inicial del profesorado, el aprendizaje de categorías centrales de la historia y la educación para la ciudadanía y la didáctica de las ciencias sociales.

En el mes de julio del año 2015 el profesor Joan Pagès visitó la Universidad Católica Silva Henríquez en el marco de un convenio entre esta casa de estudios y la Universidad Autónoma de Barcelona.

Esta entrevista busca indagar en los conocimientos del profesor Pagès como una contribución a las actuales reformas curriculares del Programa de Pedagogía en Historia y Geografía de la Escuela de Educación en Historia y Geografía de la Universidad Católica Silva Henríquez.

1. RHyG: El sistema educativo chileno imparte en conjunto las materias correspondientes a las disciplinas de historia y geografía. Sin embargo, recurrentemente se tratan como áreas de estudio independientes; ¿cómo considera usted que deben relacionarse estas disciplinas en la formación de profesores?

JP: En España los estudiantes de magisterio vienen desde secundaria con los estudios hechos en un contexto de disciplinas separadas aunque forman parte de una misma área de conocimiento, las ciencias sociales. Cuando uno se plantea cómo debería ser el currículum escolar y, en consecuencia, la formación del profesorado, uno ahí ve que el conocimiento estrictamente disciplinario cada vez funciona menos, sobre todo para una ciudadanía que tiene muchos problemas que no son disciplinarios, son problemas sociales, medioambientales, políticos, etc. Nuestra apuesta hoy pasa por un currículum

Chilena. Máster en Docencia Universitaria, Universidad de Sevilla. Académica de la Escuela de Educación en Historia y Geografía, Universidad Católica Silva Henríquez. E-mail:ncontreras@ucsh.cl 
de la formación de docentes más cercana a problemas sociales relevantes. Se trata de definir muy bien el problema y estudiar cuáles son las aportaciones de las disciplinas para su análisis y comprensión y, si hubiera lugar, su solución. Este enfoque puede dar más o menos peso a una disciplina o a otra, puede proyectarse desde una perspectiva geohistórica, puede partir del presente y desarrollar un enfoque retrospectivo, etc.

\section{RHyG: ¿Cómo se evidencian los problemas sociales relevantes en el aula?}

JP: Estando atentos a la realidad, a la vida de nuestro alumnado, a lo que ocurre en la calle y en el mundo. En la calle hay geografía, historia y todas las evidencias de cualquier disciplina social. La historia está en la calle a través de los monumentos, de la memoria de las personas. En la calle hay gente con problemas, los padres y las madres de nuestro alumnado, la ciudadanía.

El fracaso de la enseñanza se produce porque nos dedicamos a un contenido que se relaciona poco con la realidad. En mi opinión, y no es una idea original mía -ya lo decía, por ejemplo, el gran pedagogo y pensador John Dewey-, la geografía y la historia escolar deben ser el medio para entender la vida y resolver los problemas que nos presenta. Entonces debemos dar vuelta a esta situación. El conocimiento escolar es una construcción para la escuela y para la sociedad, no para la geografía y la historia académica ni tampoco para satisfacer las necesidades de los políticos.

Si esto lo cambiamos y lo interpretamos desde la perspectiva de un conocimiento que ha de permitir a la gente interpretar su mundo, las causas de los problemas y distinguir cuál es la solución, el sentido del conocimiento geográfico e histórico escolar.

\section{RHyG: Las diferentes áreas disciplinares de las ciencias sociales entran en debate por su presencia en el currículum escolar. En Chile hay una discusión en torno a la escasa presencia de la geografía como contenido mínimo obligatorio, también sobre la falta de efectividad de los contenidos transversales, como la formación ciudadana o la educación ambiental. ¿Qué opina usted en torno a estos debates?}

JP: La transversalidad fue, en España, un gran fracaso. Era una buena iniciativa para tratar los grandes problemas de la humanidad, sin embargo al no vincularse explícitamente con ninguna disciplina y al no tener el profesorado formación para desarrollarla, pronto se convirtió en "papel mojado" o en objeto de los discursos con muy poco impacto en las prácticas. Otra cosa es el papel de las disciplinas y su relación con los problemas sociales. Aquí el debate es otro. Frente a los cambios curriculares -se supone que para 
mejorar la formación de la ciudadanía- el profesorado lo primero que mira es cómo ha quedado su asignatura. Se pregunta: "qué hay de lo mío". Y argumenta: "sin geografía no se entiende el mundo, sin física no se entiende el mundo, sin...". En decir, intenta justificar el valor educativo del saber que domina. Pero el currículum tiene unas limitaciones y no cabe todo por más importante que sea. Es España, por ejemplo, la filosofía prácticamente ha desaparecido de la enseñanza obligatoria. Y no se ha hundido el mundo. Los y las docentes, el profesorado de didáctica, el profesorado universitario de historia, geografía, etc., deberíamos ser hábiles y adelantarnos a los cambios curriculares si queremos preservar nuestras enseñanzas. Deberíamos hacerlo pensando no tanto en términos corporativistas -la defensa a ultranza de nuestras disciplinas- sino en términos de los saberes necesarios para formar en la escuela obligatoria a ciudadanos y ciudadanas que serán cualquier cosa en la vida menos historiadores o geógrafos. La escuela obligatoria no forma ni geógrafos ni historiadores, sino ciudadanos y ciudadanas que, como tales, necesitan usar los saberes geográficos e históricos para ubicarse en el mundo -como electores, consumidores, productores, hombres o mujeres...-, para comprender lo que sucede y poder intervenir en su mundo con conocimiento de causa. El historiador francés Pierre Vilar nos dijo hace años a un grupo de jóvenes maestros cuando le preguntamos por qué los jóvenes tenían que aprender historia: "para leer el periódico", es decir para ubicarse en el mundo. Este debería ser el sentido de la defensa de los conocimientos escolares sociales, geográficos e históricos. Pensar más en el uso social de los mismos y menos en los saberes concretos que emanan de la academia sin los cuales, por otro lado, no podríamos ubicarnos en el mundo ni intervenir en él.

Este es un cambio bastante radical en el pensamiento del profesorado. Supone más colaboración entre los formadores de profesores, y la búsqueda de equilibrios para optimizar los saberes sociales, geográficos e históricos. En caso contrario -si seguimos peleando entre nosotros- estos saberes irán reduciendo su importancia curricular y no pasará nada, como no ha pasado nada en los países en los que desapareció de la enseñanza obligatoria el latín, la filosofía y otras asignaturas que, en algún momento, se consideraron fundamentales para la formación de los niños y de las niñas, de la juventud.

\section{RHyG: Otro debate constante en las carreras de pedagogía es la presencia de las prácticas profesionales dentro de la malla curricular. ¿Considera usted apropiado que las prácticas se incorporen desde las fases iniciales del proceso formativo?}

JP: Cuando comienzan la carrera los estudiantes vienen con una determinada imagen de lo que significa ser profesor. Esta imagen ha sido construida a lo largo de su etapa como alumnos. Hay que partir de ella para que los futuros y las futuras docentes sepan cuál es su racionalidad, de dónde parten 
profesionalmente. En un buen proceso de formación profesional la dialéctica teoría-práctica es fundamental. Hemos de formar a los futuros y a las futuras docentes a partir de problemas prácticos, no a partir de discursos. Si esta relación teoría-practica no cuadra bien y si no se convierte en la base de los problemas que se utilizan en la universidad desde el comienzo, la formación fracasa pues no logra impactar en las representaciones que el alumnado ha construido de la profesión durante su larga etapa como alumno o alumna. Y serán estas representaciones las que dirigirán sus pasos en la práctica y no los saberes que les hemos enseñado en la universidad. Esto ayuda a comprender por qué la enseñanza de las ciencias sociales, de la geografía y de la historia es tan resistente al cambio.

\section{RHyG: En ese contexto, ¿qué mecanismo considera usted el más apropiado para el desarrollo de las prácticas en el área docente?}

JP: Tal vez uno de los principales problemas de las prácticas docentes actuales es el del rol de los estudiantes ante ellas. Pueden suceder dos cosas. Las dos preocupantes. Una es que se dejan absorber por la vida en el aula, por un conjunto de cosas que idealizan porque las identifican muy bien: los niños y las niñas, la vida en el aula, etc... Es decir, todo aquello que les recuerda su época de alumnos. Y no sean capaces de tomar la distancia que les ha de permitir ver "su aula" desde otra perspectiva: la de futuros profesionales. La otra preocupación tiene que ver con los "discursos construidos" en la universidad y el papel del docente. A menudo, en lugar de observar al profesor del aula y aprender de él se dedican a juzgarlo o calificarlo. Yo no apruebo ni una cosa ni la otra. Lo que busco es que aprendan a observar y a interpretar el trabajo docente desde una perspectiva profesional, que lo contextualicen, que se pongan en el lugar del o de la docente, que colaboren con él o con ella, que observen a los y a las estudiantes para conocerles y poder decidir cómo mejor ayudarles, etc.

Deben reflexionar sobre cómo el profesor actúa frente al contexto en que trabaja, porque el trabajo docente se produce en un contexto social micro: la procedencia de los alumnos, sus familias, su situación económica, la situación de sus padres, etc. Por otro lado, cada vez más nuestros alumnos disponen de recursos tales como las computadoras, los celulares, las tablets, etc... que les hacen creer que ya lo saben todo o casi todo. La tarea docente es la consecuencia, también, de una política macro: una presión institucional, un currículum, la propia formación docente, etc... Todas esas cosas que deben tenerse en cuenta y los estudiantes en práctica deben ser capaces de observarlas, evidenciarlas, analizarlas e interpretarlas porque serán claves para su desarrollo profesional y para sus propias prácticas.

Los futuros y las futuras docentes han de aprender a partir de situaciones prácticas a analizar la racionalidad de su trabajo, de las decisiones que toman en relación con los contenidos que enseñan, con la manera como los enseñan 
y quieren que su alumnado los aprenda, con los materiales que buscan para enseñar o para aprender, con la evaluación. Han de ser capaces de justificar el uso social de los saberes que enseñan y los propósitos de su enseñanza más allá de lo que dice el currículum o los proyectos educativos de los centros. Los futuros y las futuras docentes han de ser conscientes de los modelos en los que se ubican: en el conductismo, en el constructivismo piagetiano, en el constructivismo social. Han de saber identificar la naturaleza epistemológica de los saberes que enseñan. Han de saber que sus prácticas son altamente ideológicas y han de asumir este hecho de manera crítica y creativa.

En definitiva, lo que yo les pido a los estudiantes cuando van a la práctica es que no lleguen, se sienten en el fondo del aula y miren lo que hace el profesor o sus alumnas, sino que interactúen con él, que averigüen qué estilo de enseñanza tiene, por qué usa ese estilo, que intenten interpretar y comprender lo que observan, y no tanto juzgar.

La investigación didáctica nos dice que las prácticas suelen ser muy conservadoras. Y este es otro problema a añadir a los dos anteriores. Cuando llega un joven a hacer su práctica, se le dice que olvide todo lo que aprendió en la universidad porque no le sirve. Yo creo que en las prácticas hay que ayudar a los muchachos a racionalizar, a aprender de los problemas y de las soluciones reales. Por todas estas razones, y por la necesidad de una formación más racional y crítica de los futuros docentes, la formación en didáctica de las ciencias sociales, de la geografía y de la historia de la Universidad Autónoma de Barcelona la realizamos durante muchos años en aulas de centros educativos. Tuvimos la fortuna de poder contar con la colaboración de centros y de profesores que pusieron a disposición sus aulas para que el profesorado de didáctica enseñara a enseñar a los futuros docentes enseñando. Luego en la Universidad analizábamos los resultados, preparábamos conjuntamente con los y las estudiantes las futuras intervenciones, etc. No sé si esta situación es generalizable. Sí sé que fue de las mejores experiencias en las que he participado. Lamentablemente también mi universidad consideró que era más importante la formación teórica que este modelo de formación práctico-teórico y en unos de los cambios de planes de estudio desapareció y ya no ha vuelto ni veo posibilidades de que vuelva en el futuro inmediato, sino todo lo contrario.

\section{RHyG: ¿Es compatible un estilo de educación problematizadora de los contenidos con un sistema educativo que presiona a los profesores para la obtención de buenos resultados en pruebas estandarizadas?}

JP: Esto es una realidad a nivel internacional. Se pretende enseñar ciertos valores y así evitar que la gente piense por sí misma. Es la lógica del neoliberalismo y del capitalismo global. La mejor manera de evitar que 
la gente piense por su cuenta es que rindan cuenta de lo que se pretende que deban saber.

Hoy en prácticamente todo el mundo se ha demostrado que en el aprendizaje de la historia es más relevante el contenido procedimental que el contenido factual. Es decir, que el alumnado finalice su escolaridad sabiendo analizar, argumentar, comparar, etc., más que memorizar hechos y más hechos desde la prehistoria hasta la actualidad.

En cambio, los exámenes estandarizados, como el SIMCE, se basan más en contenidos factuales con los que los gobiernos buscan comparar cómo está el alumnado de su país con respecto al de otros países que realizan pruebas similares. Lo que buscan los gobiernos es saber si el alumnado de mi país está más arriba o más abajo en relación con los resultados de los exámenes.

La perversión de evaluar la calidad de manera estandarizada, es que se focaliza en los resultados, se pone sólo el énfasis en la fase final de un proceso que es mucho más complejo. Los gobiernos deberían dar recursos económicos para mejorar la calidad durante el proceso de enseñanza, para pagar mejores sueldos a su profesorado a fin de que programase y enseñase en función del contexto y de su alumnado. ¿Por qué da tan buenos resultados el modelo de Finlandia? Porque el gobierno se dio cuenta que era importante la educación. Por lo tanto, el problema de la escuela no es un problema de los resultados de la prueba SIMCE, es un problema del presupuesto nacional del Estado. Cuando los gobiernos de todos los países concedan un $40 \%$ del fondo fiscal a la educación, las cosas, no les quepa duda, serán mucho mejor.

Por tanto, yo creo que hay alternativas. Es cierto que se presiona duramente al profesorado y se le responsabiliza de los males del sistema. Los profesores no son mártires ni deben serlo pero deben luchar para que se les reconozca su profesionalismo y deben intentar, a pesar de todo, asumirlo en su práctica cotidiana.

\section{RHyG: En el contexto de los actuales rediseños curriculares de las carreras de pedagogía que migran hacia un modelo de formación por competencias, ¿cómo se produce el acuerdo entre el área del contenido disciplinar de la historia y la geografía con la forma de evidenciar ese conocimiento disciplinar?}

JP: Esta respuesta no es nada fácil porque nos planteamos el uso social del conocimiento histórico, nos planteamos cómo los jóvenes ciudadanos y ciudadanas han de usar el conocimiento histórico en contextos de vida que no son los de la profesión de historiador, sino que son los de cualquier tipo de actividad en cualquier contexto social y laboral, que puede ser tan amplio y heterogéneo como son nuestras sociedades. Por lo tanto, ¿cómo la historia 
ayuda a una chica que decide ser mecánica de automóviles o un chico que decida ser cocinero, o a un chico y a una chica que deciden ser aviadores?, ¿Qué uso hemos de dar al conocimiento histórico, geográfico y social en cualquiera de nuestros ámbitos de vida? El uso social del conocimiento histórico consiste, en mi opinión, en ayudar a los ciudadanos y ciudadanas a desarrollar un pensamiento histórico y social para sentirse en la historia, es decir, para sentirse miembros de una sociedad en la que continuamente deberán tomar decisiones porque el pasado les ha legado una historia determinada, un mundo determinado, que es en el que están y en el que van tomando decisiones para construir progresivamente su futuro personal y social.

En este sentido, cabe preguntarse, ¿qué competencias debería desarrollar el alumnado para entender la historicidad del presente? Yo creo que las mismas que han desarrollado los historiadores pero con una salvedad: nuestros alumnos y nuestras alumnas no serán historiadores. En su vida cotidiana podrán utilizar productos históricos (novela y cine histórico, museos y lugares de memoria, yacimientos, etc...). Pero en su vida cotidiana no se encontrarán con problemas históricos, se van a encontrar con problemas. Algunos serán económicos, otros ambientales, culturales, religiosos, vecinales, laborales, de cualquier tipo. Se van a encontrar con problemas que tienen unas causas, unos antecedentes y que deberán comprender para poder participar en su solución. Para resolver estos problemas hemos de dotarles de un pensamiento histórico, geográfico y social que les permita saber por qué las cosas son como son y les permita argumentar sus interpretaciones sobre las cosas que han sido, son y serán. Es decir, la escuela y la enseñanza de la historia, de la geografía y de las ciencias sociales ha de dotar al alumnado de un pensamiento histórico, geográfico y social fundamental para entender lo que ocurre en su mundo. La ciudadanía ha de salir de la escuela con un pensamiento temporal y una conciencia histórica que les permita comprender la relación pasado, presente y futuro. Con un pensamiento causal, que le ayude a averiguar que las cosas no ocurren porque sí. Un pensamiento que les permita preguntar a las fuentes primarias qué nos dicen sobre el pasado, un pensamiento que ayude a elaborar relatos y narrativas para expresar sus ideas sobre lo que ha sido o es, un pensamiento que ayude a empatizar con los demás. Y, por supuesto, que les ayude a tomar decisiones éticas frente a determinados acontecimientos del pasado, del presente y del futuro.

Fundamentalmente la enseñanza de la historia y de las ciencias sociales debe ayudar a los y a las jóvenes ciudadanas a desarrollar un pensamiento que les ayude a emitir juicios de valor con conocimiento de causa. Es decir, un pensamiento ético que les capacite para emitir juicios ante cualquier circunstancia. Ante las reivindicaciones mapuches, si son justas; ante los conflictos que podemos tener con otros países limítrofes, ante las cuestiones económicas que hacen más profunda la desigualdad social, ante la situación que está sucediendo con las migraciones en el Mediterráneo desde África, ante las tragedias humanas que estamos viviendo, ante las atrocidades del Estado Islámico y ante 
cualquier hecho que, por activa o por pasiva se nos presente y nos obligue a tomar decisiones. Yo creo que las cosas deberían ir en esta dirección.

\section{RHyG: Respecto de la didáctica, en el currículum basado en la enseñanza por competencias, es muy importante lo que van a hacer los profesores universitarios; ¿cómo perci- be usted la formación de profesores de historia, geografía y ciencias sociales, desde la didáctica universitaria, es decir, del formador de formadores?}

JP: Ese es uno de los actuales problemas que tenemos en la formación de profesores. Yo diría que mi modelo de profesor se ubica en esto que se llama la práctica reflexiva, por lo tanto, en una formación inicial en la que los futuros docentes han de ser educados y formados para ir tomando decisiones. Quizás uno de los principales problemas que tenemos hoy es que la universidad sigue practicando básicamente una enseñanza transmisiva, por lo tanto, en la que los futuros profesores toman pocas decisiones.

Me gustaría pensar que podríamos concretar todo esto en modelos de formación en los que la relación teoría práctica no fuese la actual, en la que primero damos la teoría y luego van a la práctica a comprobar las virtudes de la teoría, y si la práctica no funciona como funciona la teoría, la práctica no es buena. Lo mejor es avanzar hacia una práctica reflexiva. Y para ello no se puede predicar a nuestros futuros y futras profesoras lo que tendrán que hacer en los colegios cuando enseñen, sino que hemos de practicarlo en nuestras aulas universitarias (evidentemente, no se trata de reproducir los mismos modelos metodológicos que en la escuela básica o media sino de dar protagonismo a quienes aprenden, acercándoles a los problemas de la enseñanza y del aprendizaje de una manera mucho más dialéctica). Durante muchos años, la formación en didáctica de la historia y de las ciencias sociales de los estudiantes de magisterio y de profesorado de secundaria de la Universidad Autónoma de Barcelona se hacía en aulas de colegios a cargo de los propios profesores de didáctica. En la Universidad analizábamos con los y las estudiantes que habían asistido a nuestras clases la información recogida, los problemas que nos habíamos encontrado y buscábamos alternativas a los mismos. Y con ellos volvíamos a la práctica.

\section{RHyG: ¿Cuál es la responsabilidad social que tiene la historia} y la geografía desde la pedagogía? ¿son disciplinas que deberían validar los procesos políticos o ampliar una visión crítica de estos?

JP: La pregunta es muy interesante. Saber para qué se enseña historia, cuáles son los propósitos de la enseñanza de la historia, ayuda mucho a comprender qué historia se enseña y por qué se enseña la historia que se enseña. 
Si partimos del origen de la enseñanza de esta disciplina veremos que desde la fundación de la escuela pública la historia tiene como misión crear conciencia identitaria nacional y, en consecuencia, intentar orientar el futuro de la ciudadanía. En este sentido, no han cambiado mucho las cosas desde que se funda la escuela hasta hoy. Hoy sigue siendo interés preferente de todos los políticos y todos los gobiernos pensar que la historia es el conocimiento que ha de permitir a los ciudadanos y ciudadanas formar su identidad, una identidad que tiene mucho más que ver con la manera como la interpretan los poderes gobernantes que no con lo que una democracia plural y diversa debería enfatizar.

El tipo de historia que se está enseñando en buena parte del mundo es una historia política fundamentalmente eurocéntrica y androcéntrica, en la cual sólo se da una interpretación -la de nuestros triunfadores-cuando la misma historia como disciplina ha generado paradigmas distintos, ha permitido interpretaciones distintas a los diferentes acontecimientos y se ha abierto a estudiar todo lo humano y a todos y todas sus protagonistas. Sin embargo, la concepción de la historia escolar dominante en los currículos y en las prácticas se ubica más en el campo positivista que en el de cualquier otro paradigma historiográfico. Por ello se sigue estudiando lo que se ha estudiado siempre, se sigue haciendo de la misma manera y, en consecuencia, la historia sigue hablando de cosas y personajes alejados de la vida de nuestro alumnado y, en consecuencia, la mayor parte de los jóvenes ciudadanos y ciudadanas que están aprendiendo historia, no saben del papel que en nuestro pasado y en nuestro presente han tenido las mujeres, los niños y las niñas, los indígenas, los pobres, los homosexuales, etc.

Por lo tanto, la razón de ser de la enseñanza de la historia y de las ciencias sociales no ha de ser socializar en los valores hegemónicos sino contrasocializar. Ha de ser una historia que ayude a los jóvenes a pensarse a sí mismos dentro de la historia, una historia que apueste por una ciudadanía democrática en la que no necesariamente lo que otros dicen hay que creerlo sin más, como si fuera un dogma, sino que hay que saber interpretarlo a partir de los argumentos y contrastarlo con todo tipo de fuentes.

\section{RHyG: ¿Qué sugeriría usted a los profesores, para lograr la formación de ciudadanos responsables?}

JP: Yo cada vez estoy más convencido de que ser profesor hoy es una profesión de alto riesgo. Primero, porque está compitiendo con otros campos del saber que hace unos años no existían, porque la escuela ya no es la única entidad que transmite conocimiento Tenemos competidores mucho más potentes. Los mismos chicos y chicas, desde jovencitos, manejan el celular con el que pueden acceder a mucha información. Manejan sin problemas tablets, internet, se mueven por las redes, por los canales de televisión, etc. 
La situación ha cambiado radicalmente desde cuando la escuela era la única entidad que acercaba el conocimiento a la ciudadanía.

Hoy ser profesor o profesora es una profesión de riesgo que se puede superar tranquilamente si uno asume que es un intelectual comprometido y que ha de ejercer una actividad que se caracteriza fundamentalmente por tomar decisiones continuamente. Es decir, si uno o una cree que su tarea no se limita a hacer seguidismo de aquello que otros han decidido que hay que enseñar, o de los manuales, sino que toma decisiones en función de una población única e irrepetible que son los niños y las niñas, los y las jóvenes que tiene en sus aulas. Se debe apostar por ser un profesional reflexivo, es decir, que toma decisiones en función del grupo humano que tiene delante, que es capaz de saber las aspiraciones que tienen sus muchachos y muchachas y de ayudarles a construir su propio pensamiento, un pensamiento histórico y social que les va a permitir ubicarse en su mundo, leer su realidad, tomar decisiones en esa realidad $y$, en definitiva, ser un ciudadano reflexivo que es capaz de participar con los demás, en profundizar en la democracia, en construir una convivencia democrática que responda a nuestras necesidades actuales y a la justicia social.

En este caso, la historia y la geografía son dos variables fundamentales, saber dónde estamos y por qué estamos donde estamos, de dónde venimos y sobre todo hacia dónde nos gustaría ir. La enseñanza de la historia es siempre una apuesta de futuro, aunque la historia enseña el pasado; en realidad la intencionalidad de los políticos es que enseñe el pasado porque quieren construir un determinado tipo de futuro. La apuesta del y de la docente es dar las herramientas a los y a las jóvenes para que construyan el futuro que quieran construir, no el que otros deciden imponerles. 